\title{
Intralipid Increases Lung Polyunsaturated Fatty Acids and Protects Newborn Rats from Oxygen Toxicity
}

\author{
ILENE R. S. SOSENKO, SHEILA M. INNIS, AND LEE FRANK \\ Divisions of Neonatology and Pulmonary Research, Calvin and Flavia Oak Asthma Research Facility, \\ Departments of Pediatrics and Medicine, University of Miami School of Medicine, Miami, Florida, 33101 \\ [I.R.S.S., L.F.] and Department of Pediatrics, University of British Cohumbia. \\ Vancouver, Canada V5Z4H4 [S.M.I.]
}

\begin{abstract}
Intralipid, derived from soybean oil and containing a high percentage of $n-6$ family polyunsaturated fatty acids (PUFA) and also linolenic acid, an n-3 family PUFA, is commonly the first fat source provided to very low birth weight premature infants. Following up on our previous reports that newborn rats born to dams fed highPUFA diets demonstrate superior tolerance to hyperoxia, we examined whether the high-PUFA fat source Intralipid might also protect against oxygen toxicity. Adult female rats were fed either regular Rat Chow or fat-free diet containing $20 \%$-Intralipid as the fat source for $3 \mathrm{wk}$ before and then throughout pregnancy and lactation. One- and 5d-old offspring of Intralipid diet-fed dams demonstrated significant increases in lung lipid n-6 family PUFA plus elevated linolenic acid compared with regular diet-fed offspring. These characteristic fatty acid patterns, apparent in total lung lipids, were even more pronounced in the triglyceride fraction compared with the phospholipid fraction. Associated with these fatty acid changes were significantly improved hyperoxic survival rates (89 out of $95=94 \%$ survival after $7 \mathrm{~d}$ of $>95 \% \mathrm{O}_{2}$ exposure) in Intralipid offspring (versus 89 out of $106=84 \%, p<0.05$ in regular diet offspring) and evidence of superior clinical/ pathologic status. No differences in pulmonary antioxidant enzyme or surfactant system development, response of antioxidant enzymes to hyperoxic exposure, or lung prostaglandin $E_{2}$, 6-keto $P G F_{1}-\alpha$ or leukotrienes $C_{4}-F_{4}$ were present. These findings continue to support the hypothesis that increasing lung PUFA content may provide increased $\mathrm{O}_{2}$ free radical scavenging capacity, thus protecting against hyperoxic lung damage. The results also suggest a role for Intralipid administration in protecting the lungs of high oxygen-exposed very low birth weight premature infants. (Pediatr Res 30: 413-417, 1991)
\end{abstract}

\section{Abbreviations}

PUFA, polyunsaturated fatty acids

VLBW, very low birth weight

SOD, superoxide dismutase

CAT, catalase

GP, glutathione peroxidase

DSPC, disaturated phosphatidylcholine

$\mathrm{PGE}_{2}$, prostaglandin $\mathrm{E}_{2}$

Received March 1, 1991; accepted July 3, 1991

Correspondence and reprint requests: llene R. S. Sosenko, M.D., Department of Pediatrics (R-131), University of Miami School of Medicine, P.O. Box 016960, Miami FL 33101

Supported in part by March of Dimes Basic Research Grant (I.R.S.S.), Career Investigatorship for the British Columbia Children's Hospital (S.M.I.), and Ross Laboratories.
6-keto-PGF $1-\alpha$, 6-keto-prostaglandin $\mathrm{F}_{1}-\alpha$

$\mathrm{LTC}_{4}-\mathrm{F}_{4}$, leukotrienes $\mathrm{C}_{4}-\mathrm{F}_{4}$

$\mathrm{BPD}$, bronchopulmonary dysplasia

$\mathrm{P} / \mathrm{S}$, polyunsaturated/saturated fatty acid ratio

With the progression of modern neonatal intensive care, greater numbers of VLBW oxygen- and ventilator-requiring premature infants are surviving. However, despite favorable outcomes in terms of mortality, these infants are at extremely high risk of developing the chronic lung process known as BPD, considered to be related, at least in part, to the damaging effect of oxygen on the lung (1). Because of the overwhelming clinical problems that these VLBW premature infants experience in the first several days of life, nutritional needs are frequently not the highest priority in their care. In fact, common nursery practices consist of a several-day interval between the birth of a VLBW oxygen-requiring premature infant and the initial administration of lipid nutrition. Because of the vulnerability of the VLBW premature infant to the effects of a delay in lipid nutrition (2) and the possible role that lipids might play in protecting the lung from oxygen toxicity, the early withholding of lipid nutrition from these critically ill neonates might increase their risk of developing the chronic lung disease and damage of BPD.

Evidence is available from the literature to suggest that lung lipid composition is related to hyperoxic tolerance and protection against hyperoxic lung damage in experimental animals. We have performed a series of studies in newborn rats that has demonstrated that lipid nutrition, containing high concentrations of PUFA, confers protection against pulmonary oxygen toxicity. Specifically, newborn rat offspring of dams fed diets high in PUFA demonstrated elevated concentrations of PUFA in their lung lipids and significantly improved survival in hyperoxia compared with offspring of dams fed regular Rat Chow; conversely, newborn offspring of dams fed low PUFA, high saturated fatty acid diets were found to be most susceptible to pulmonary oxygen toxicity $(3,4)$. The improved hyperoxic tolerance of newborn rats was found to be more or less constant with qualitatively different high PUFA diets (i.e. high n-6 family PUFA diet containing safflower oil and high n-3 family PUFA diet containing menhaden fish oil). With these studies as background and in an attempt to use the newborn experimental animal to examine a common nutritional intervention of the human premature infant, we have now tested whether a highPUFA diet based on Intralipid (the commercially available initial fat preparation administered to premature infants, containing high $n-6$ family PUFA but also considerable amounts of the $n-3$ fatty acid, linolenic acid) would produce substantial increases in 
lung PUFA content and provide a meaningful degree of protection against $\mathrm{O}_{2}$ toxicity in newborn rats.

\section{MATERIALS AND METHODS}

Animals and diets. Adult Sprague-Dawley albino female rats $(\sim 200 \mathrm{~g})$ were fed one of two diets ad libitum: $l)$ standard Rat Chow (no. 5001; Ralston-Purina, Co., St. Louis, MO) [5\% fat as a mixture of animal and vegetable fats, containing $27 \%$ linoleic acid $(18: 2 \mathrm{n} 6), 3 \%$ linolenic acid $(18: 3 \mathrm{n} 3), \mathrm{P} / \mathrm{S}=1.0]$; or 2$)$ Intralipid diet ["fat-free" test diet no. 15750; United States Biochemical Corp., Cleveland, $\mathrm{OH}$, plus $10 \%$ lipid (by weight), using 20\%-Intralipid (KabiVitrum, Inc., Alameda, CA) as the fat source, containing $50 \%$ linoleic acid (18:2n6), 9\% linolenic acid $(18: 3 \mathrm{n} 3), \mathrm{P} / \mathrm{S}=4.4]$. The two diets were essentially comparable in protein and carbohydrate content. Rats were fed the respective diets for at least $3 \mathrm{wk}$ before breeding and then throughout pregnancy and lactation. Breeding was accomplished by placing male and female animals together overnight, checking for spermpositive vaginal smears the following morning, and considering the midpoint of the cohabitation period as the onset of pregnancy. Newborn rats were nursed exclusively, thereby receiving rat milk from respective diet-fed dams.

Several litters from the two diet groups were killed prematurely at 20 or $21 \mathrm{~d}$ of gestation, after term delivery at $22 \mathrm{~d}$ of gestation, or at 1 or $5 \mathrm{~d}$ of postnatal age. Premature pups were delivered by hysterotomy under pentobarbital anesthesia; newborn rats were obtained after normal parturition (either within $6 \mathrm{~h}$ of delivery of the first pup or after $24 \mathrm{~h}$ or $5 \mathrm{~d}$ of life). Fetal, newborn, 1- or 5-d-old rat pups were killed with an overdose of pentobarbital, and their lungs were perfused immediately in situ via the pulmonary artery using cold saline. The perfused fetal and newborn lungs were removed, stripped of nonpulmonary tissue, and homogenized in 20-30 times their weight of cold saline in a Brinkmann polytron (high speed, $90 \mathrm{~s}$ ) (Brinkmann Instruments, Inc., Westbury, NY). For preterm and term newborns, two to four lungs were pooled and homogenized to obtain adequate tissue for the antioxidant enzyme and phospholipid analyses. One-d-old and 5-d-old rat pup lungs were utilized for fatty acid analyses (as described below).

Antioxidant enzyme, phospholipid, and fatty acid analyses. Aliquots of lung homogenate were analyzed for SOD (5), CAT (6), and GP (7) activities and for DNA (8) and protein (9) content using standard spectrophotometric techniques. Purified standards for SOD, CAT, and DNA assays were obtained from Sigma Chemical (St. Louis, MO) and GP was obtained from Boehringer-Mannheim (Houston, TX). Results of antioxidant enzyme analyses are expressed as activity units/mg DNA (and also calculated per mg protein and per g lung). For analyses of lung tissue DSPC content, separate aliquots of lung homogenates were lipid extracted using the method of Bligh and Dyer (10), reacted with osmium tetroxide according to Mason (11), and assayed for inorganic phosphorus as described by Morrison (12). A known quantity of ${ }^{14} \mathrm{C}$-dipalmitoyl-phosphatidylcholine (New England Nuclear, Boston, MA) was added before lipid extraction, and aliquots were counted at each step to estimate and correct for sequential losses. DSPC was expressed as $\mathrm{mg} / \mathrm{g}$ lung weight.

Lungs from 1-d-old and 5-d-old rat offspring from both diet groups were perfused with iced saline as described above, and then frozen in liquid nitrogen in preparation for fatty acid analyses. The lung tissue was subsequently homogenized, aliquoted for protein [assayed according to the method of Lowry (13)], and then subjected to lipid extraction using the procedure of Folch (14). Total fatty acids were converted to their respective methyl esters with methanolic $\mathrm{HCl}$ and separated and quantitated by capillary column gas-liquid chromatography (15). C17:0 was used as the internal standard. The fatty acid composition of phospholipid and triglyceride lipid subclasses was determined after separation of phospholipids from the other lipid classes by plate chromatography (16).
Exposure to hyperoxia. Multiple litters (nine to 11 pups/litter) of newborn rats from the two diet groups were placed with their dams into $>95 \% \mathrm{O}_{2}$ (or room air) at 6-12 h of life. Details of the continuous 7-d exposure conditions have been previously reported (3). Dams from the same diet group were rotated between room air and $>95 \% \mathrm{O}_{2}$-exposed litters every $24 \mathrm{~h}$ to prevent poor mothering resulting from $\mathrm{O}_{2}$-induced maternal illness. Weight gain and hyperoxic survival of newborn rats from each diet group were recorded daily for $7 \mathrm{~d}$. In addition, after an exposure period of $7 \mathrm{~d}$, random surviving offspring of both diet groups were removed from hyperoxia and room air-exposed litters, were killed, and their lungs then perfused, homogenized, and assayed for DSPC and antioxidant enzyme activities as described above. Using separate litters of $>95 \% \mathrm{O}_{2}$-exposed newborns from the two diet groups, we evaluated the clinicopathologic status after $7 \mathrm{~d}$ of hyperoxic exposure. Pups were scored in a blinded fashion by two investigators, and received a score of 0 (normal), $0.5,1.0$ (moderately abnormal), 1.5 , or 2 (markedly abnormal) on each of five parameters of $\mathrm{O}_{2}$ toxicity: degree of respiratory distress, pleural fluid quantitation, and gross appearance of lung edema, atelectasis, and lung hemorrhage. Potential range of total clinicopathologic score was 0 to 10 .

In a separate experiment, offspring from the two diet groups were killed after $5 \mathrm{~d}$ of either room air or $>95 \% \mathrm{O}_{2}$ exposure, and their lungs were removed and homogenized as described above, with the homogenizing buffer containing acetylsalicylic acid $(15 \mathrm{mM})$. Fresh homogenates were assayed for 6-keto-PGF $\alpha, \mathrm{PGE}_{2}$, and $\mathrm{LTC}_{4}-\mathrm{F}_{4}$ using specific RIA test kits (Seragen, Inc., Cambridge, MA).

Statistics. Statistics were performed using $\chi^{2}$ analysis for survival data and unpaired $t$ test for fatty acid, antioxidant enzyme, disaturated phosphatidylcholine, and clinicopathologic data. When multiple $t$ tests were performed (i.e. with fatty acid, antioxidant enzyme, and DSPC data), a Bonferoni-type correction was made, allowing as significant a $p$ value of less than 0.01 (17).

\section{RESULTS}

Growth and biochemical development of the offspring of the two diets. The Intralipid-based diet was well accepted by the female rats. Maternal weight, fetal weight and number, and neonatal growth were not different between the Intralipid experimental diet group and the group fed regular Rat Chow.

Antioxidant enzymes, surfactant, and fatty acid analyses. The comparative late gestational development of the pulmonary antioxidant enzyme and surfactant systems in the experimental Intralipid diet and regular diet fetuses and newborns revealed no significant differences between the two diet groups in SOD, CAT, or GP activities or in lung DSPC content during gestational d $20-22$, the final $3 \mathrm{~d}$ of gestation.

Table 1 shows the major fatty acid components of total lung lipid in 1-d and 5-d-old offspring of the Intralipid diet and regular diet groups. Significant elevations in linoleic acid $(18: 2 n 6)$, linolenic acid (18:3n3), and total n-6 family PUFA were present in the 1-d-old Intralipid group and, by $5 \mathrm{~d}$ of age, most of these fatty acid differences were more pronounced and included an unexpected significant decrease in arachidonic acid (20:4n6) and in total n-3 family PUFA in the Intralipid offspring.

Analysis of the fatty acid composition of the triglyceride fraction and the phospholipid fraction of total lung lipid in $1-\mathrm{d}$ and 5-d-old offspring of the two diet groups can be seen in Table 2. In the lungs of the 1-d-old offspring, those of the Intralipid group demonstrated PUFA elevations in the two lipid fractions compared with the regular diet group. However, the fatty acid composition of the lung triglyceride fraction in 1-d-old Intralipid offspring versus regular offspring demonstrated more marked elevations in $18: 2 \mathrm{n} 6,20: 4 \mathrm{n} 6$, total n-6 family PUFA, and total PUFA compared with the fatty acid differences seen in the phospholipid fraction. By $5 \mathrm{~d}$ of life, the elevations in $18: 2 \mathrm{n} 6$, 
Table 1. Major fatty acid components of total lung lipid in 1-and 5-d-old offspring of two diet groups*

\begin{tabular}{|c|c|c|c|c|c|c|c|c|}
\hline & $16: 0$ & $18: 2 \mathrm{n} 6$ & $18: 3 n 3$ & $20: 4 n 6$ & $\begin{array}{r}\text { Total } \\
\text { PUFA } \\
\end{array}$ & $\begin{array}{l}\text { Total } \\
n-6\end{array}$ & $\begin{array}{l}\text { Total } \\
\mathrm{n}-3\end{array}$ & $\mathrm{P} / \mathrm{S}$ \\
\hline \multicolumn{9}{|l|}{$1-d$} \\
\hline Intralipid & 39 & $8 \dagger$ & $0.25 \dagger$ & 11 & 30 & $24 \dagger$ & 5 & 0.6 \\
\hline Regular & 39 & 5 & 0.09 & 10 & 27 & 19 & 7 & 0.5 \\
\hline \multicolumn{9}{|l|}{$5-d$} \\
\hline Intralipid & 35 & $16 \dagger$ & $0.64 \dagger$ & $7 \dagger$ & 34 & 29 & $5 \dagger$ & 0.7 \\
\hline Regular & 29 & 7 & 0.11 & 13 & 34 & 25 & 8 & 0.7 \\
\hline
\end{tabular}

* Values are expressed as $\%$ of total and are means; $n=4-8$ lung samples/group. Fatty acids: 16:0, palmitic acid; 18:2n6, linoleic acid; 18:3n3, linolenic acid; $20: 4 \mathrm{n} 6$, arachidonic acid.

$\dagger p<0.01$ or less between diet groups.

Table 2. Major fatty acid components of triglyceride and phospholipid fractions of lung lipid in 1 - and 5-d-old offspring of diet groups*

\begin{tabular}{|c|c|c|c|c|}
\hline & \multicolumn{2}{|c|}{ Triglyceride } & \multicolumn{2}{|c|}{ Phospholipid } \\
\hline & Intralipid & Regular & Intralipid & Regular \\
\hline \multicolumn{5}{|l|}{ 1-d-old } \\
\hline $18: 2 \mathrm{n} 6$ & $15 \dagger$ & 10 & $8 \dagger$ & 6 \\
\hline $18: 3 n 3$ & 0.51 & 0.37 & $0.34 \dagger$ & 0.17 \\
\hline $20: 4 \mathrm{n} 6$ & $6 \dagger$ & 4 & 14 & 13 \\
\hline Total PUFA & $38 \dagger$ & 29 & 32 & 28 \\
\hline Total $n-6$ & $30 \dagger$ & 20 & 26 & 21 \\
\hline Total $n-3$ & 6 & 8 & 6 & 7 \\
\hline $\mathrm{P} / \mathrm{S}$ & 1.0 & 0.7 & 0.6 & 0.5 \\
\hline \multicolumn{5}{|l|}{ 5-d-old } \\
\hline $18: 2 n 6$ & $27 \uparrow$ & 12 & $11 \dagger$ & 7 \\
\hline $18: 3 n 3$ & $1.3+$ & 0.4 & $0.4 \uparrow$ & 0.2 \\
\hline $20: 4 n 6$ & $6 \dagger$ & 4 & 16 & 18 \\
\hline Total PUFA & $51 \dagger$ & 41 & 37 & 38 \\
\hline Total $n-6$ & $41 \dagger$ & 26 & 31 & 30 \\
\hline Total $n-3$ & $8 \dagger$ & 13 & $5 \dagger$ & 8 \\
\hline $\mathrm{P} / \mathrm{S}$ & $1.8 \dagger$ & 1.2 & 0.8 & 0.9 \\
\hline
\end{tabular}

* Values are $\%$ of total and are means; $n=4-8$ lung samples/group. $\dagger p<0.01$ or less between diet groups.

18:3n3, total n-6 family PUFA, total PUFA, and P/S and the depression in total n-3 family PUFA in the Intralipid versus regular groups had become even more pronounced in the triglyceride compared with the phospholipid fraction of lung lipids.

Exposure to hyperoxia. Offspring of Intralipid diet dams demonstrated significantly superior survival ( 89 out of $95=94 \%$ ) during $7 \mathrm{~d}$ of $>95 \% \mathrm{O}_{2}$ exposure compared with regular diet offspring ( 89 out of $106=84 \%$ ), $p<0.05$. In addition, Intralipid offspring showed evidence of superior clinical/pathologic status after $7 \mathrm{~d}$ of hyperoxic exposure (Table 3), with an average clinicopathologic score of $1.56 \pm 0.14$ compared with an average score of $2.55 \pm 0.25$, for regular diet offspring exposed to hyperoxia for $7 \mathrm{~d}(p<0.05)$. [Note that the high survival rate and relatively low clinical/pathologic score in regular diet newborns is a reflection of the innate relative $\mathrm{O}_{2}$ tolerance of neonatal versus adult animals. For adults in $>95 \% \mathrm{O}_{2}$, the comparable survival rate would be $\sim 10-20 \%$ and the clinical/pathologic score in the survivors $\sim 7-9(18)]$.

The improved 7-d hyperoxic survival in Intralipid offspring was not associated with a significantly improved capacity to mount a protective antioxidant enzyme response to hyperoxia compared with regular diet offspring (Table 4).

Lung tissue levels of $\mathrm{PGE}_{2}, 6$-keto-PGF $-\alpha$, and $\mathrm{LTC}_{4}-\mathrm{F}_{4}$ in 5-d-old offspring continuously exposed to either room air or $>95 \% \mathrm{O}_{2}$ since birth are shown in Table 5. No significant differences were found in baseline (room air) levels of these eicosanoids, nor in levels after $5 \mathrm{~d}$ of hyperoxic exposure in the two diet groups.

\section{DISCUSSION}

Intralipid is a commercial fat emulsion designed for i.v. administration. It is derived primarily from soybean oil, with the addition of glycerin and egg yolk phospholipids as emulsifiers. This fat preparation (or its equivalent from other commercial sources), containing a generous amount of the n- 6 PUFA linoleic acid $(50 \%)$ and the $n-3$ PUFA linolenic acid (9\%), represents the initial fat source provided to most VLBW premature infants to ensure adequate caloric intake and prevent essential fatty acid deficiency before the onset of oral feedings. As a logical followup to our previous nutritional studies $(3,4)$, we chose to examine in the newborn rat the effect of this common nutritional intervention in terms of influencing lung fatty acid patterns and tolerance to high oxygen exposure. We utilized Intralipid as the total fat source added to the fat-free experimental diet that was fed to rat dams before and throughout pregnancy and lactation [We had previously determined that rat milk reflects quite closely the fatty acid composition of the diet of the rat dam $(3,4)]$. Oned-old offspring of dams fed the Intralipid diet demonstrated increased linoleic and linolenic PUFA in lung lipids; the 5-d-old offspring of Intralipid diet dams demonstrated more marked increases in lung PUFA than 1-d-old offspring, suggesting the importance not only of the intrauterine fatty acid milieu but also a substantial postnatal dietary influence as well. The most striking difference in lung PUFA enrichment in the Intralipid diet rat pups was noted in the triglyceride (nonmembrane) lipid fraction as opposed to the phospholipid (membrane-associated) lipid fraction.

The outcome of the present study using Intralipid is consistent with our previous findings indicating that increasing lung lipid PUFA (especially the triglyceride fraction) of newborn rat offspring is associated with significant protection against hyperoxic lethality and the pathologic effects of high oxygen exposure on the lung $(3,4)$. Whereas previous investigations examined saf-

Table 3. Clinicopathological (CP) score of offspring of Intralipid and regular diet rats after $7 d$ in $>95 \%$ oxygen*

\begin{tabular}{ccccccc}
\hline Diet group & $\begin{array}{c}\text { Clinical } \\
\text { status }\end{array}$ & $\begin{array}{c}\text { Pleural } \\
\text { fluid }\end{array}$ & Edema & Hemorrhage & Atelectasis & $\begin{array}{c}\text { Total CP } \\
\text { score } \dagger\end{array}$ \\
\hline Intralipid & 0.53 & 0.12 & 0.54 & 0.20 & 0.17 & $1.56 \pm 0.14 \dagger$ \\
Regular & 0.27 & 0.35 & 0.75 & 0.35 & 0.82 & $2.55 \pm 0.25$ \\
\hline
\end{tabular}

* Values are mean values for regular $(n=20)$ and intralipid $(n=30)$. Possible scores for each item: $0=$ normal; $0.5,1=$ moderately abnormal; 1.5, $2=$ markedly abnormal. Possible total score: $0-10$.

$\dagger$ Mean \pm SEM

$\ddagger p<0.05$ between diet groups. 
Table 4. Response of antioxidant enzymes and surfactant (DSPC) after exposure to $7 d>95 \% \mathrm{O}_{2}$ in Intralipid and regular diet rat pups*

\begin{tabular}{lcccc}
\hline & \multicolumn{3}{c}{ Activity units/mg DNA } & DSPC (mg/g \\
\cline { 2 - 5 } & SOD & CAT & GP & lung) \\
\hline Air-Intralipid diet & $13.2 \pm 0.8$ & $238 \pm 36$ & $0.29 \pm 0.07 \dagger$ & $3.01 \pm 0.17$ \\
O $_{2}$-Intralipid diet & $18.9 \pm 1.3$ & $476 \pm 81$ & $0.98 \pm 0.23$ & $4.65 \pm 0.29$ \\
& $\uparrow 43 \%$ & $\uparrow 100 \%$ & $\uparrow 238 \%$ & $\uparrow 55 \%$ \\
Air-regular diet & $13.3 \pm 1.0$ & $262 \pm 54$ & $0.63 \pm 0.11$ & $3.74 \pm 0.42$ \\
O $_{2}$-regular diet & $17.4 \pm 2.3$ & $442 \pm 45$ & $1.40 \pm 0.14$ & $4.72 \pm 1.92$ \\
& $\uparrow 30 \%$ & $\uparrow 69 \%$ & $\uparrow 123 \%$ & $\uparrow 26 \%$ \\
\hline
\end{tabular}

* Mean values $\pm 1 \mathrm{SD}$ and $\%$ change for $\mathrm{O}_{2}$ group $v s$ air group values after 7 -d exposure to $>95 \% \mathrm{O}_{2}$ in Intralipid and regular diet groups; $n=$ 4 lung samples/group; all enzyme values after hyperoxia are significantly higher than air values in both diet groups.

$\dagger p<0.01$; baseline (air) value for GP is significantly lower in the Intralipid group; it is because of this that the response of GP to hyperoxia in the Intralipid group is significantly greater than hyperoxic response of GP in regular group.

flower oil (high n-6 PUFA oil) and menhaden fish oil (high n-3 PUFA oil), the present study using Intralipid examined the effect of a preparation high in both $n-6$ family PUFA (linoleic acid) and n-3 PUFA (linolenic acid). Thus, a third high-PUFA fat source has now been shown to confer hyperoxic protection of essentially similar magnitude as the other tested high-PUFA diets (i.e. $94 \%$ survival after $7 \mathrm{~d}$ of $>95 \% \mathrm{O}_{2}$ for Intralipid versus $95 \%$ for safflower oil versus $91 \%$ for menhaden fish oil) compared with the 7-d hyperoxic survival rate for regular diet rats, which has ranged from 67 to $84 \%$.

The present and previous studies from our laboratory are among several reported in vivo investigations linking unsaturation of lung lipids to improved hyperoxic tolerance (18-20). In addition, our present in vivo investigation is in agreement with the findings of a recent in vitro study using rabbit tracheal epithelial cells. These cells, when cultured in lipid-supplemented media, demonstrated increased PUFA content and increased hyperoxic viability (21).

The mechanism by which PUFA lipid enrichment works to confer hyperoxic protection on the lung is not fully known. Results from this study and previous ones do not indicate that elevated PUFA work through accentuating the development of the pulmonary antioxidant enzymes or surfactant production, nor through enhancing the ability of the newborn animal to mount a protective increase in pulmonary antioxidant enzymes in response to hyperoxic exposure. Other investigators have suggested a role for lung eicosanoids in the development of (or protection from) oxygen toxicity (22-25). In our present study, we were unable to demonstrate differences in $\mathrm{PGE}_{2}$, 6-keto$\mathrm{PGF}_{1}-\alpha$ (the metabolite of prostacyclin), or in the $\mathrm{LTC}_{4}-\mathrm{F} 4$ in lung tissue of either diet group either in air or hyperoxia (Table 5). In addition, in our previous studies, we found a similar degree of protection against pulmonary $\mathrm{O}_{2}$ toxicity in newborn offspring of rat dams fed either a safflower oil-based or fish oil-based highPUFA diet, even though the safflower diet pups demonstrated significantly elevated lung eicosanoids whereas the fish oil diet pups had significantly decreased lung eicosanoid levels compared with regular diet pups $(3,4)$. Our studies thus strongly suggest that eicosanoids do not appear to be playing a major role in the increase in hyperoxic tolerance found in the Intralipid offspring.

Having shed doubts on the role that antioxidant enzymes, surfactant, and lung eicosanoids might be playing in the hyperoxic protection of Intralipid offspring, results of our present (and previous) studies suggest that the increased PUFA content in lung tissue itself may, at least in part, be an explanation for the consistently observed increased oxygen tolerance in our neonatal rats. Dormandy (26) has proposed, in a theory diametrically opposite to traditional biochemical theory, that intracellular PUFA, located in noncritical, nonmembrane sites, could serve to avidly scavenge excess $\mathrm{O}_{2}$ free radicals and thereby prevent their toxic interaction with critical membrane PUFA, thus protecting cells from hyperoxic or $\mathrm{O}_{2}$ free radical-mediated damage. Our lung fatty acid data from the Intralipid (versus regular diet) offspring are consistent with the hypothesis of Dormandy because in the present study (and our previous ones) hyperoxic protection was clearly associated with increases in total PUFA and $\mathrm{P} / \mathrm{S}$ of the lung triglyceride (intracellular, nonmembrane) lipid fraction in the high PUFA diet offspring. Other possible mechanisms by which Intralipid nutrition and the resultant increased lung PUFA might influence cellular tolerance to high $\mathrm{O}_{2}$ exposure, such as by alterations in membrane enzyme systems (27), membrane permeability (28), or membrane fluidity (29) have not been explored in the present investigation.

Our present findings of increased hyperoxic protection of newborn experimental animals associated with Intralipid nutrition raises questions as to whether the early administration of Intralipid to VLBW oxygen-requiring premature infants (during their period of maximum need for supplemental oxygen) might provide protection against the damaging effects of oxygen on their lungs. The likely immaturity of their antioxidant defense systems (e.g. antioxidant enzymes, vitamins $\mathrm{E}$ and $\mathrm{A}$ ) would tend to make these VLBW prematures extremely vulnerable to lung damage associated with high $\mathrm{O}_{2}$ therapy. Any antioxidant advantage that might be derived from early nutritional intervention might make a difference in lessening the susceptibility of these premature newborns to the development of chronic lung disease or BPD.

The results of two clinical studies using Intralipid treatment, each with 20 babies or less per group, have appeared in the recent literature $(30,31)$. One study demonstrated that early postnatal lipid supplementation was associated with a decreased incidence of severe BPD (30), but the other suggested that lipid therapy during the 1st wk of life might actually increase the risk of developing chronic lung disease (31). However, the results from this latter study have been questioned because of apparent differences in the initial severity of respiratory distress in the control versus Intralipid-treated group (32). More extensive and larger scale investigations will be necessary to clarify this disparity in clinical outcome. Nonetheless, our findings of protection by Intralipid against pulmonary $\mathrm{O}_{2}$ toxicity expand on our previous studies linking high-PUFA dietary treatment with hyperoxic protection, and add further impetus to the continued investiga-

Table 5. Lung tissue prostaglandin and leukotriene levels in 5-d-old Intralipid and regular diet offspring exposed to $>95 \% \mathrm{O}_{2}$ or air*

\begin{tabular}{lccc}
\hline \multicolumn{1}{c}{ Group } & 6-keto PGF $-\alpha$ & PGE $_{2}$ & LT C $_{4}-\mathrm{F}_{4}$ \\
\hline Room air exposed & & & \\
$\quad$ Intralipid & $19160 \pm 1640$ & $35350 \pm 2950$ & $18190 \pm 5250$ \\
$\quad$ Regular diet & $17140 \pm 2160$ & $33750 \pm 2950$ & $14090 \pm 4270$ \\
$>95 \% \mathrm{O}_{2}$ exposed & $18240 \pm 2500$ & $34810 \pm 4510$ & $7220 \pm 1930 \dagger$ \\
$\quad$ Intralipid & $20700 \pm 3380$ & $31830 \pm 6090$ & $6200 \pm 2910 \dagger$ \\
$\quad$ Regular diet &
\end{tabular}

* $\mathrm{pg} / \mathrm{g}$ lung; $n=8 / \mathrm{group}$; no differences between the two diet groups are statistically significant.

$\dagger p<0.01: \mathrm{O}_{2}$-exposed groups $v s$ respective air groups. 
tion of the role that early clinical use of Intralipid might play in protecting the vulnerable oxygen-requiring VLBW infant from oxygen toxicity and the development of the chronic lung process of BPD.

Acknowledgments. The authors thank Miguel Martinez and Laurie Nicol for their expert technical support.

\section{REFERENCES}

1. Bancalari E, Sosenko I 1990 Pathogenesis and prevention of neonatal chronic lung disease: recent developments. Pediatr Pulmonol 8:109-116

2. Farrell PM, Gutcher GR, Palta M, DeMets D 1988 Essential fatty acid deficiency in premature infants. J Clin Nutr 48:220-229

3. Sosenko IRS, Innis SM, Frank L 1988 Polyunsaturated fatty acids and protection of newborn rats from oxygen toxicity. J Pediatr 1 12:630-637

4. Sosenko IRS, Innis SM, Frank L 1989 Menhaden fish oil, n-3 polyunsaturated fatty acids and protection of newborn rats from oxygen toxicity. Pediatr Res 25:399-404

5. McCord JM, Fridovich I 1969 Superoxide dismutase: an enzymatic function for erythrocuprein (hemocuprein). J Biol Chem 244:6049-6055

6. Holmes RS, Masters CJ 1970 Epigenetic interconversion of the multiple forms of mouse liver catalase. FEBS Lett 11:45-48

7. Paglia DE, Valentine WN 1967 Studies in the quantitative and qualitative characterization of erythrocyte glutathione peroxidase. I Lab Clin Med 70: $158-159$

8. Richards GM 1974 Modifications of the diphenylamine reactions giving increased sensitivity and simplicity in the estimation of DNA. Anal Biochem 57:360-376

9. Schacterle GR, Pollack RL 1973 A simplified method for the quantitative assay of small amounts of protein in biological material. Anal Biochem 51:654-655

10. Bligh EF, Dyer WJ 1959 A rapid method of total lipid extraction and purification. Can J Biochem Physiol 37:911-917

11. Mason RJ, Nellenbogen J, Clements JA 1976 Isolation of disaturated phosphatidylcholine with osmium tetroxide. J Lipid Res 17:281-284

12. Morrison WR 1964 A fast, simple and reliable method for the microdetermination of phosphorus in biological materials. Anal Biochem 11:218-224

13. Lowry OH, Rosebrough NJ, Farr AL, Randall RJ 1951 Protein measurement with folin reagent. J Biol Chem 193:265-275

14. Folch J, Lees M, Sloane-Stanley GH 1957 A simple method for the isolation and purification of total lipids from animal tissues. 3 Biol Chem 226:497509
15. Hrboticky N, Mackinnon MJ, Innis SM 1990 Effect of a linoleic acid-rich vegetable oil formula on tissue fatty acid accretion in the brain, liver, plasma and erythrocytes of infant piglets. Am J Clin Nutr 51:173-182

16. Innis SM, Clandinin MT 1981 Dynamic modulation of mitochondrial innermembrane lipids in rat heart by dietary fat. Biochem J 193:155-167

17. Fisher RA 1970 Statistical Methods for Research Workers. Hafner Press, New York, pp 213-224

18. Yam J, Frank L, Roberts RJ 1978 Oxygen toxicity: comparison of lung biochemical responses in neonatal and adult rats. Pediatr Res 12:115-119

19. Clements JA 1971 Comparative lipid chemistry of lungs. Arch Intern Med 127:387-389

20. Kehrer JP, Autor AP 1978 The effect of dietary fatty acids on the composition of aduit rat lung lipids: relationship to oxygen toxicity. Toxicol Appl Pharmacol 44:423-430

21. Dennery PA, Kramer CM, Alpert SE 1990 Effect of fatty acid profiles on the susceptibility of cultured rabbit tracheal epithelial cells to hyperoxic injury. J Respir Cell Molec Biol 3:137-144

22. Stenmark KR, Eyzaguirre M, Westcott JY, Henson PM, Murphy RC 1987 Potential role of lipid mediators of inflammation in bronchopulmonary dysplasia. Am Rev Respir Dis 136:770-772

23. Burghuber OC, Atrife RJ, Zirrolli J, Henson PM, Henson JE, Mathias MM, Reeves JT, Murphy RC, Voelkel NF 1985 Leukotriene inhibitors attenuate rat lung injury induced by hydrogen peroxide. Am Rev Respir Dis 131:778785

24. Farrukh IS, Michael JR, Peters SP, Sciuto AM, Adkinson AF, Freeland HS, Paky A, Spannhake EW, Summer WR, Gurtner GH 1988 The role of cyclooxygenase and lipoxygenase mediators in oxidant-induced lung injury. Am Rev Respir Dis 137:1343-1349

25. Smith LU, Sommers E, Hunt CE, Pachman L 1986 Hyperoxic lung injury in mice: a possible protective role for prostacyclin. J Lab Clin Med 108:479488

26. Dormandy TL 1969 Biological rancidification. Lancet 2:684-688

27. Houslay MD 1985 Regulation of adenylate cyclase activity by its lipid environment. Proc Nutr Soc 44:157-165

28. Henning B, Shasby DM, Fulton AB, Spector AA 1984 Exposure to free fatty acid increases the transfer of albumin across cultured endothelial monolayers. Arteriosclerosis 4:489-497

29. Storch J, Schacter D 1984 Dietary induction of acyl chain desaturases alters the lipid composition and fluidity of rat hepatocyte plasma membranes. Biochemistry 23:1165-1170

30. Perlman M, Fabrizio M 1990 Effect of postnatal lipid nutrition on long-term outcome of children with bronchopulmonary dysplasia. Am Rev Respir Dis 141:A158(abstr)

31. Hammerman C, Aramburo MJ 1988 Decreased lipid intake reduces morbidity in sick premature neonates. J Pediatr 113:1083-1088

32. Frank L, Sosenko IRS, Bancalari E, Chen C 1989 Early intralipid and bronchopulmonary dysplasia. [letter] J Pediatr 115:658-659 